\title{
KNOWLEDGE-BASED EVALUATION OF PART ORIENTATION DESIRABILITY IN POWDER BED FUSION ADDITIVE MANUFACTURING
}

\author{
Mbow, Mouhamadou Mansour (1); \\ Marin, Philippe René (1); \\ Perry, Nicolas (2); \\ Vignat, Frédéric (1); \\ Grandvallet, Christelle (1) \\ 1: Univ. Grenoble Alpes, CNRS, Grenoble INP, G-SCOP, 38000 Grenoble, France; \\ 2: Univ. Bordeaux, CNRS, Arts et Métiers, I2M, Esplanade Arts Et Métiers, 33405 Talence, France
}

\begin{abstract}
In powder bed fusion (PBF) additive manufacturing, the definition of part orientation is one of the most important steps as it affects the quality, the cost and the build time of products. Different works already attempted to propose methodologies for the assessment of optimal build orientation based on criteria such as the minimization of support volume. Elicitation works with industry experts have shown that they use much more varied rules to determine the orientation of parts. For instance, they do not treat the different surfaces of the part the same way (e.g., experts state that "priority surfaces of the part must be oriented close to vertical"). Today, the available tools do not allow integrating these kind of specifications. This paper discusses a knowledge-based methodology for the evaluation of part candidate orientations in PBF. Desirability function approach is used to translate companies' expertise in the form action rules into mathematical functions that are tested on geometries to provide metrics for assisting the decision-making. A case study is presented to illustrate the use of this desirability function approach on complex part orientation problem.
\end{abstract}

Keywords: Additive Manufacturing, Decision making, Action rule, Desirability function, Knowledge management

Contact:

Mbow, Mouhamadou Mansour

Grenoble Institute of Technology

GSCOP Laboratory

France

mouhamadou-mansour.mbow@grenoble-inp.fr

Cite this article: Mbow, M. M., Marin, P. R., Perry, N., Vignat, F., Grandvallet, C. (2021) 'Knowledge-Based Evaluation of Part Orientation Desirability in Powder Bed Fusion Additive Manufacturing', in Proceedings of the International Conference on Engineering Design (ICED21), Gothenburg, Sweden, 16-20 August 2021. DOI:10.1017/pds.2021.457 


\section{INTRODUCTION}

In powder bed fusion (PBF) additive manufacturing (AM), after the design phase, multiple steps are necessary to prepare the part (orientation, definition of the regions to support, design of the support structures, nesting etc.) before the production. Among these, the orientation selection step appears to be one of the most significant one (Leutenecker-Twelsiek et al. (2016)). The orientation of a given part can be defined by two angles $\alpha$ and $\beta$ in the global reference $(o, x, y, z)$. As shown by various research efforts, those two orientation parameters alone have a huge impact on the desired process duration (thus, the time to market), the quality expected by the customers and the production cost (Das et al. (2015), Delfs et al. (2016)). Today, it is also well known that almost all of the downstream CAM steps such as the definition of the regions to support and the nesting of multiple parts are highly affected by the chosen orientation.

Hence, to ensure the successful materialisation of a design, it is necessary to find a suitable orientation.

The optimal definition of $\alpha$ and $\beta$ requires a certain level of skills of the process in question and remains a major concern in industries despite the availability of CAM tools covering different features. Some investigations have shown that the industry practitioners usually make use of their know-how to make decisions on manufacturing parameters (Sanfilippo et al. (2019)). For part orientation, Grandvallet et al. (2020) have collected a set of particular practices from industry experts using PBF systems. For instance, they showed that experts differentiate the surfaces of the part and classify them into categories as post-machined surfaces, priority surfaces and machining datum surfaces. Each class of surface must be oriented in a specific way according to them. These knowledge assets have been translated into action rules (knowledge resources in the form of prescription to fulfil). However, it is still difficult to evaluate rules in literal form on geometries as complex as those used in AM. So far, these kind of rules are not integrated in the commercial software or in the algorithms proposed in the literature to solve part orientation problems. In the view of that, this paper discusses the integration of action rules from experts to assist the decision-making on part orientation parameters.

For a set of action rules and a part to orient, the general idea of this study is to propose metrics corresponding to the level of compliance of the action rules when applied to the part. These values intend to ease the choice of parameters. The approach of this paper is to first identify action rules used by industry experts to orient parts through a brief review, then the action rules found are quantitatively represented to enable their assessment on geometries. Finally, a case study is presented to illustrate the approach.

\section{KNOWLEDGE RESOURCES FOR PART ORIENTATION IN INDUSTRY}

The cycle of expert knowledge use for decision-making starts from the acquisition which can be either performed by elicitation (interviewing experts, Cooke (1994)) or by screening company reports, machine catalogues and brochures to capture manufacturers knowledge (Kumar et al. (2006)). Following the acquisition, different types of knowledge objects can be identified and used for various purposes (e.g. training of worker, problem solving, decision-making etc.).

For the part orientation activity, Grandvallet's work with a company using PBF systems revealed a number of knowledge resources in the form action rules. Action rules define actions that should be performed in specific time and place to reach some objectives. This type of knowledge has the ability to clearly describe an expertise in the form of prescription to fulfil. A set of action rules used by industry practitioners to define orientation for PBF parts have been reported. Six of them are used in this work as developed below.

\subsection{Minimize part shadow on start plate (AR1)}

The projected area of the part on the build platform (start plate) must be minimal to allow the maximum nesting. The extent of the shadow varies with the orientation of the geometry. Thus, the orientation is a significant factor upon the production efficiency. Indeed, the productivity in PBF is driven by the quantity of products treated simultaneously (Vayre (2014)). In electron beam melting (EBM), for instance, it takes time to prepare a build due to operations like setting the plate to the right elevation, filling the powder and preheating the chamber. As these operations need to be repeated at any production, it is more profitable to put as many parts as possible on the same batch. 


\subsection{Minimize overhanging non-machined surfaces (AR2)}

According to its topology and its orientation, a component might exhibit different overhanging zones. In AM in general, built overhanging surfaces have deformations resulting from shrinkage and warping due to residual stresses (Körner (2016)). To alter or eliminate those harmful effects, support structures are added during the manufacturing process to get the surfaces anchored and prevented from the eventual distortions (Hussein et al. (2013)). However, after their removal, those support structures will in turn leave undesired marks affecting the surface quality. To remove those marks it is usually necessary to perform machining which generates additional costs. In PBF, functional surfaces are usually machined by default (the cost and time are planned), thus, it is better orienting the part such that the support structures are built on these post-machined surfaces. This action rule prescribes then to minimize the overhanging non-machined surfaces in order to avoid support marks.

\subsection{Orient priority surfaces close to vertical (AR3)}

According to the experts, a surface is called priority when it has to be of good quality from the additive manufacturing step (here PBF); that is to say that no post-processing operation will be performed on it. Shortly speaking, a priority surface must be acceptable or good "AS IS". In order to reach a certain quality, these types of surfaces must be oriented vertically or near to the vertical, (a close to vertical range can be defined as shown in Figure 1). In the literature, experimental works led by Kranz et al. (2015) also show that the surface roughness decreases as the surface gets near to the vertical. The roughness $R a$ was approximately $10 \mu \mathrm{m}$ for $90^{\circ}$ and $30 \mu \mathrm{m}$ for $0^{\circ}$.

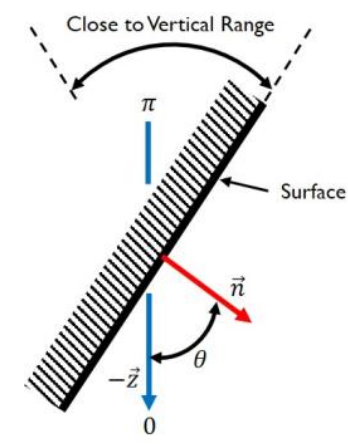

Figure 1. Priority surface illustration; close to vertical range

This action rule has different utilities. For instance, as geometries are generally complex with organic shapes or lattices, it is not obvious to perform finishing or polishing on some surfaces. First, because finding systems able to maintain or clamp the components in position is costly. Second, because the tool trajectories might be complex and require massive efforts to establish a process planning.

\subsection{Orient machining datum surfaces out of horizontal (AR4)}

As post-processing is usually required (especially the machining of functional surfaces), it is necessary for CAD engineers as well as for CAM engineers to think of the isostatic positioning. For this reason, in the part orientation activity, those surfaces must clearly be indicated; they are called machining datum surfaces. Inaccuracies linked with parallelism, perpendicularity, flatness and so on need to be avoided. Experts indicated that the machining datum surfaces should be oriented out of horizontal in order to avoid such inaccuracies.

As an illustration, refer to the cantilever part in Figure 2. The surfaces M1 and M2 are post-machined (by milling process) based on the datum surfaces R1, R2 and R3 (Figure 2a). The indicated tolerance values are from Das et al. (2015). The output part is manufactured by using EBM (Ti-6Al-4V) in the indicated orientation (Figure 2b). As a result, the surface R1 is severely deformed despite the added support structures (similar results can be found in Körner (2016) and Mugwagwa et al. (2018)). Therefore, the required perpendicularity and flatness are no longer respected. In addition, the reference surface A is a little bit bent towards the left side (Figure 2c). Machining the part basing on the datum surfaces R1, R2 and R3 would obviously result in many errors and could damage the tools used. 


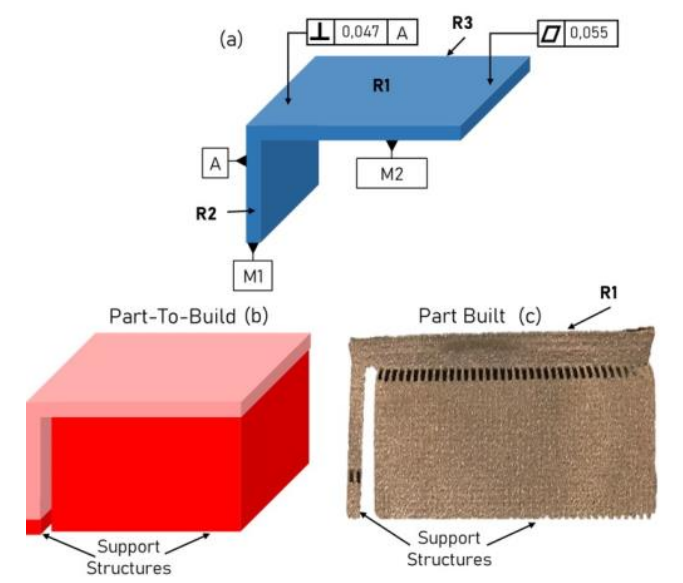

Figure 2. Illustration of machining datum orientation

\subsection{Minimize shape deformation risks (AR5)}

In PBF, if a certain number of long layers are stacked, there may be risks of progressive shrinking deformation (Vo et al. (2018)). The shrinkage usually causes loss of the lower edges of parts as illustrated in Figure 3 (the left side of the part is fixed).

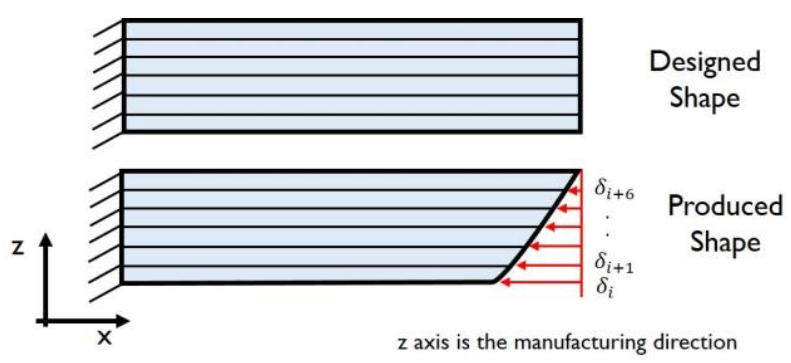

Figure 3. Loss of edge (adapted from [Vo2018])

From several experiments led by Vo et al. (2018) and Ghaoui et al. (2020) it has been inferred that the shrinkage of a layer is highly dependent on the number of layers above it. In fact, every deposited layer $i$ shrinks when solidifying and cooling. The following melted layer $i+1$ also does the same, but this time it influences the layer $i-1$ making it shrink again. This continues until the number of layers stacked below the layer $i$ is massive enough to stop the induced shrinkage. Therefore, for a layer $i$, the influence of the layer $i+1$ is greater than that of $i+2$ which is greater than that of $i+3$ and so on.

Distributing support structures highly helps preventing some surface from this issue. Used practitioners usually prefer finding orientations for which long stacked layers are avoided. Today, it is one of most efficient way to minimize this shape deviation.

\subsection{Avoid support removal difficulty on surfaces (AR6)}

This action rule means "don't put support structures on a surface if you cannot or have difficulty to remove them after manufacturing". For industry practitioners, it constitutes one of the most important criteria when defining the manufacturing orientation and designing support structures. A badly located support removal can necessitate great efforts or consume much time and can even be irremovable. For these non-profitable reasons, practitioners usually perform this analysis.

For a given surface, it is suggested that its accessibility depends on the available space in front of it. Therefore, the distances separating it from the surrounding surfaces. Figure 4 shows two examples of removal difficulty. To comply with this action rule, one of these conditions need to be satisfied: (1) the surface in question must not be overhanging or (2) there must be enough space to access this surface. 


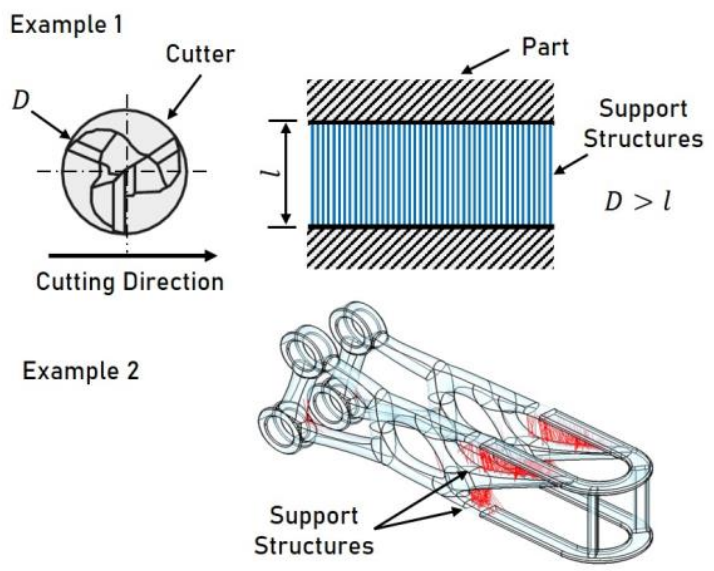

Figure 4. Example 1: Tool size greater than available space; Example 2: Removal difficulty on complex part

\section{QUANTITATIVE REPRESENTATION OF THE ACTION RULES}

Here, the quantitative representation model proposed by Mbow et al. (2021) is used. Their approach consisted of transforming action rules into desirability functions (Derringer and Suich (1980), Costa et al. (2011)). This process of transforming knowledge assets expressed in literal form into mathematical form was referred to as mathematization. The use of desirability functions make it possible to convert different variable (usually noncomparable) into dimensionless values between 0 and 1 . In this context, these values represent the compliance levels of the action rules for a given orientation. The mathematization process proposed by Mbow et al. (2021) is summarized below.

\section{- Codification}

The codification is a stage of knowledge formalization. It makes it possible to represent the concepts of the action rules by following a generic grammar. Three classes of concepts namely the part, the evaluation concepts and the actions were established. The structure of an action rule is such that an action is used to achieve an objective by acting on an evaluation concept that is measured on the part. The class part includes the different information linked with the product and its attributes. Four actions namely, minimize, maximize, avoid and require were proposed. Figure 5 shows an example of action rule codification. The objective is to get as many priority surfaces of the part as possible close to vertical, thus, the verb is replaced by one of the four actions.

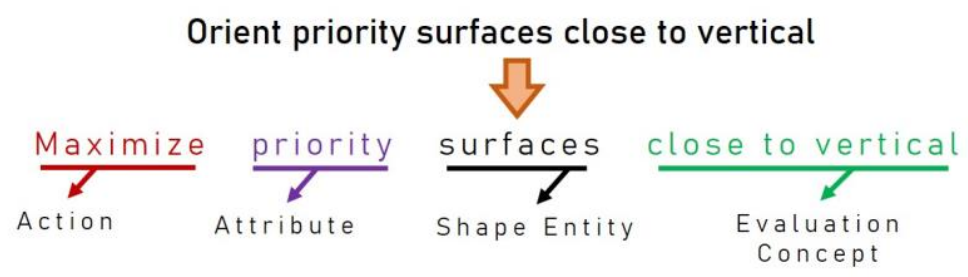

Figure 5. Example of action rule codification

\section{- Mathematization}

First, a variable of the shape entity that has a great impact on the evaluation concept is identified. For instance, the fact that a surface (shape entity) is close to vertical (evaluation concept) can be measured based on its inclination angle. Then, the way the evaluation concept evolve with respect to that identified variable is determined. For the example of priority surfaces close to vertical (AR3), this relational function linking the shape to the evaluation concept is given in Figure 6a. Finally, the desirability function is built based on the determined relational function and the action contained in the codified action rule. If the action is, maximize or require, the maximum values of the evaluation concept are searched, thus the desirability function is equal to the relational function. If the action is minimize or avoid, the desirability function is equal to the negation of the evaluation concept. For the previous example, the desirability function is given in Figure 6b. More details on the mathematization process can be found in Mbow et al. (2021). 

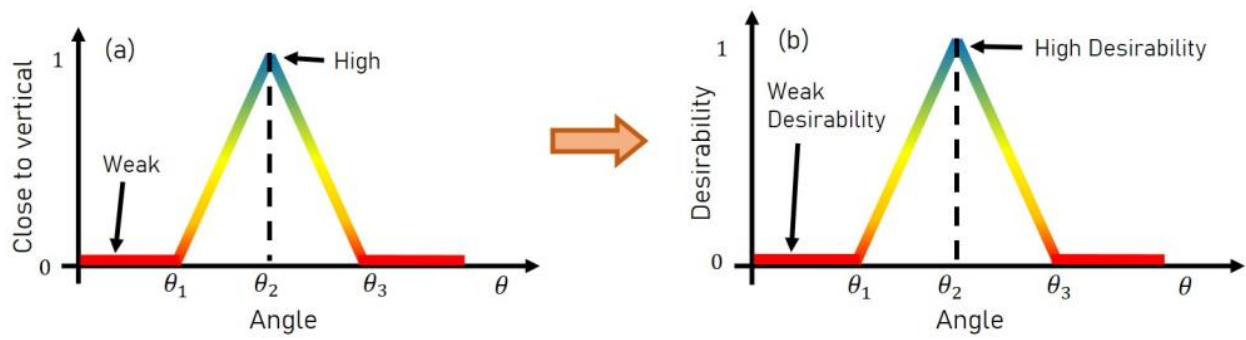

Figure 6. (a) Relational Function; (b) Desirability Function

Table 1. Mathematical representation of orientation action rules

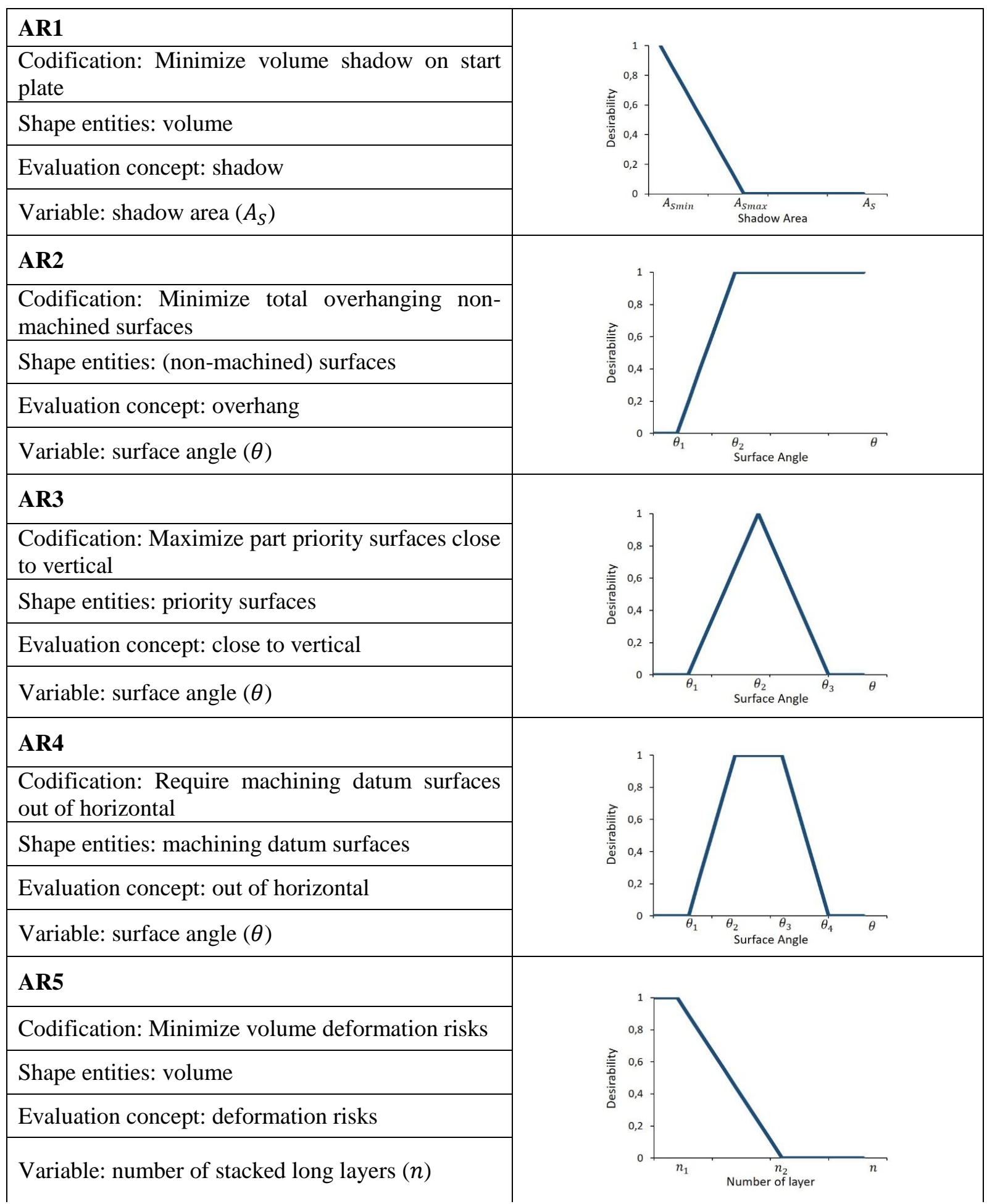




\begin{tabular}{|l|l|}
\hline AR6 \\
\hline $\begin{array}{l}\text { Codification: Avoid support structures and } \\
\text { support removal difficulty on surfaces with } \\
\text { potential support difficult to remove }\end{array}$ \\
\hline $\begin{array}{l}\text { Shape entities: surfaces with potential support } \\
\text { difficult to remove }\end{array}$ \\
\hline $\begin{array}{l}\text { Evaluation concepts: support structures, support } \\
\text { removal difficulty }\end{array}$ \\
\hline Variables: surface angle $(\theta)$, distance face/face $(d)$
\end{tabular}

\subsection{Modelling of part orientation action rules}

In this section, the mathematical models of the six action rules previously presented are provided. They are given in Table 1. The codified version of action rules, the variable for measuring the evaluation concept on the shape entities are summarized.

\subsection{Implementation}

In order to support the assessment of the part orientation desirability and the decision making process, a CAM tool called COFFA has been developed as part of this work. The COFFA tool covers different features as cited below.

- A 3D viewer to display and interact with the imported geometries (in STEP or STL format),

- A surface selection manager to enable the assignment of attributes such as machined or priority to the surfaces of the part,

- Computation algorithms for each type of evaluation concept.

Given the complexity of the geometries and evaluation concepts to be computed, it would be a difficult task to manually assess the desirability of an orientation. This tool makes it possible to compute the desirability of hundreds of orientations and gives to the decision maker a set of comparable alternative solutions displayed on response surfaces (illustration in Figure 7). For each action rule, a response surface is expected.

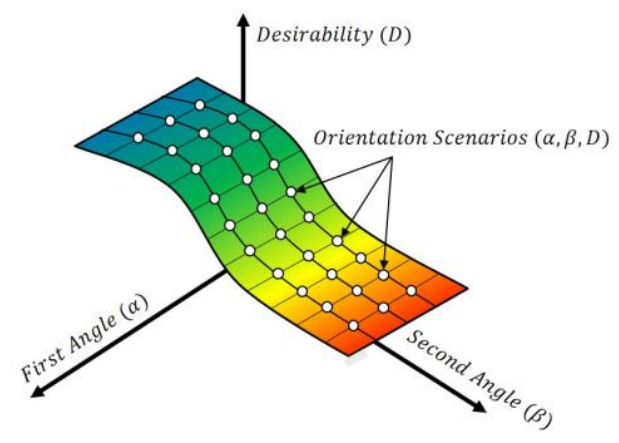

Figure 7. Response surface for part orientation desirability

\section{CASE STUDY}

This section presents an illustrative case of part orientation problem with specific requirements. The rotations $\alpha$ (around $O X$ ) and $\beta$ (around $O Y$ ) vary in the ranges $\left[0^{\circ}, 180^{\circ}\right]$ and $\left[0^{\circ}, 360^{\circ}\right]$ respectively, and by steps of $10^{\circ}$ for both. A total of 700 orientations are calculated, at each iteration, all of the rules are applied to the geometry to evaluate the desirability values. Benchmarks with a commercial software available to the team, in particular Magics (from Materialise) are performed. 


\subsection{Problem Definition}

The part of interest is an angular support for hammock. An example of positioning of this topologyoptimized shape is shown in Figure 8a. The aim is to find an optimal orientation of the part such that the action rules are compliant enough.

All the surfaces of the part are non-machined (after additive manufacturing) except those in green, which are functional surfaces. The red surfaces are also functional as the rope is in permanent contact with them. However, their post-machining might be complex and expensive; thus, they are designated as priority surfaces. This means that they must already be of good quality from the additive manufacturing stage. A dedicated algorithm computes the surfaces with potential support difficult to remove, as they change at every new orientation of the part. The action rules AR1, AR2, AR3 and AR6 are considered.

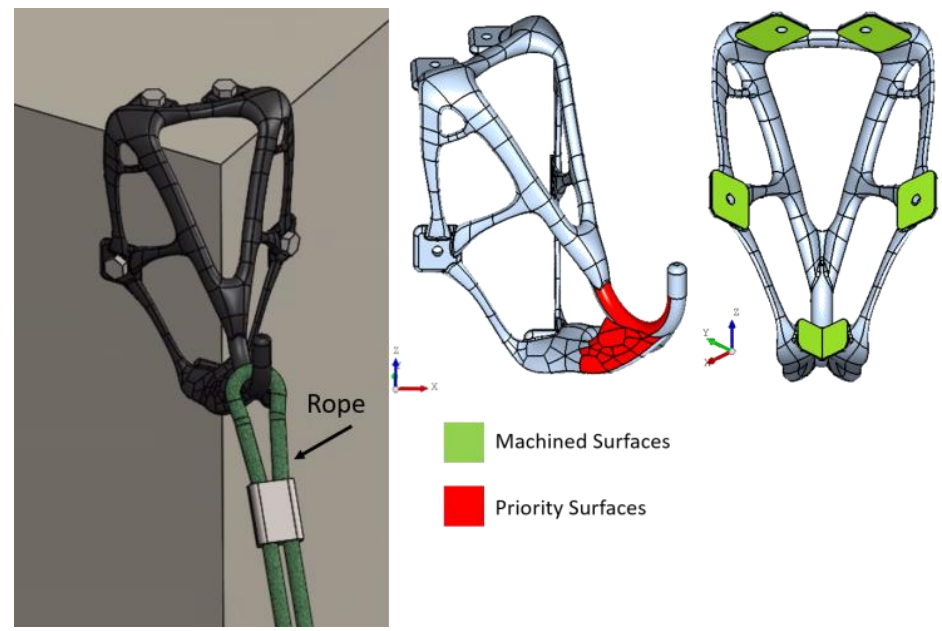

Figure 8. Hammock rope support: (a) Mounted example; (b) Part with surface attributes

\subsection{Results}

As the results show (Figure 9), for rules that allow compensation (when the action is minimize or maximize), there are several points where the desirability is high. For instance, the response surface of the AR2 that is about the overhanging non-machined surfaces. According to its corresponding desirability function, the surfaces for which the angle is greater than $50^{\circ}$ compensate those that have a poor desirability. That is also the case for AR3 that considers the verticality of the priority surfaces. However, for this action rule (AR3), the desirability values do not reach great values because the surfaces of interest are mutually perpendicular, it is necessary to find a trade-off between them. That is why, during its codification the defined action was not "require" which would result in zero desirability for almost all of the orientations. This effect can be illustrated by the action rule about the support removal difficulty for which the action is "avoid". For a set of points $(\alpha, \beta)$, the rule is not compliant at all $\left(D_{A R 6}=0\right)$. The matches of these points on the other response surfaces should be avoided at the decision making stage.

To make a choice among all these available solutions, the decision-maker must balance the action rules in order to find a good trade-off. Some examples of solutions are given in Figure 10. In the scenario $\left(0^{\circ}-0^{\circ}\right)$, the rules AR1 and AR2 have a great compliance level, but the others have low ones. For that orientation, there would be support difficult to remove. The part could be manufactured with either the orientation $\left(100^{\circ}-40^{\circ}\right)$ or $\left(90^{\circ}-230^{\circ}\right)$ to meet up the action rules prescribed by the experts. 

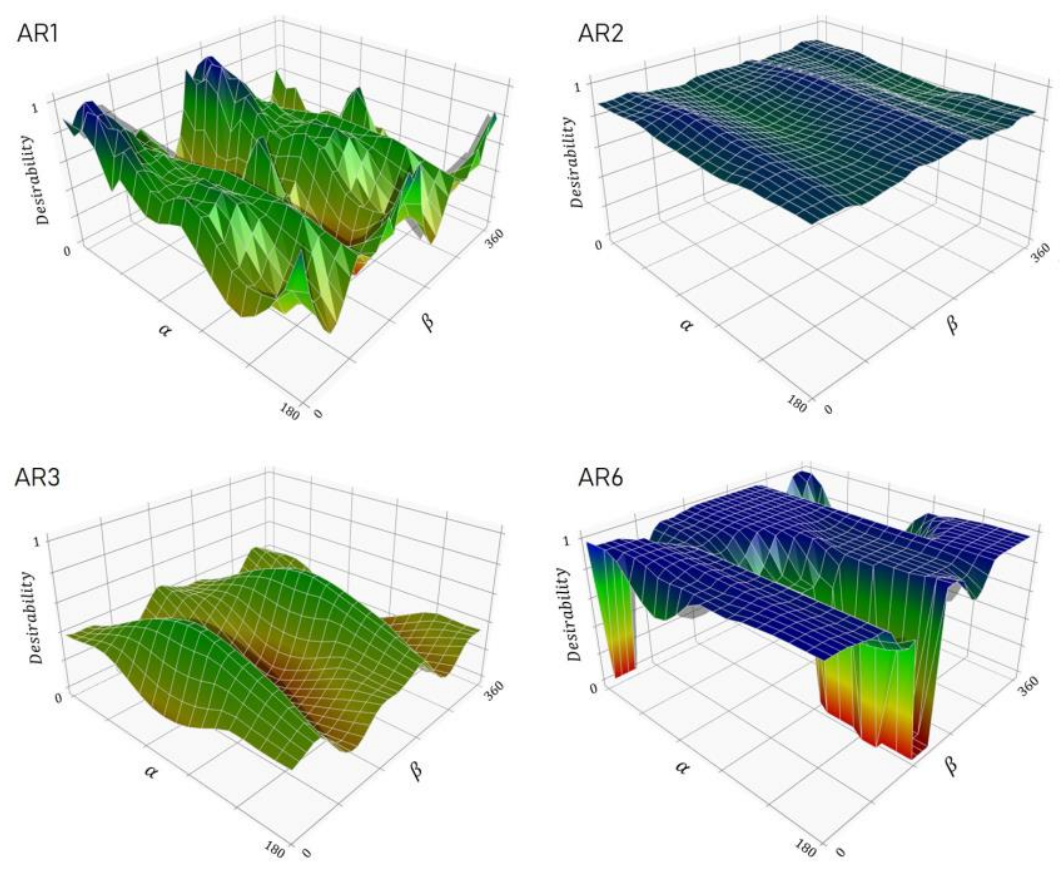

Figure 9. Response surfaces: (AR1) Part shadow on start plate; (AR2) Overhanging nonmachined surfaces; (AR3) Priority surfaces; (AR6) Support difficult to remove

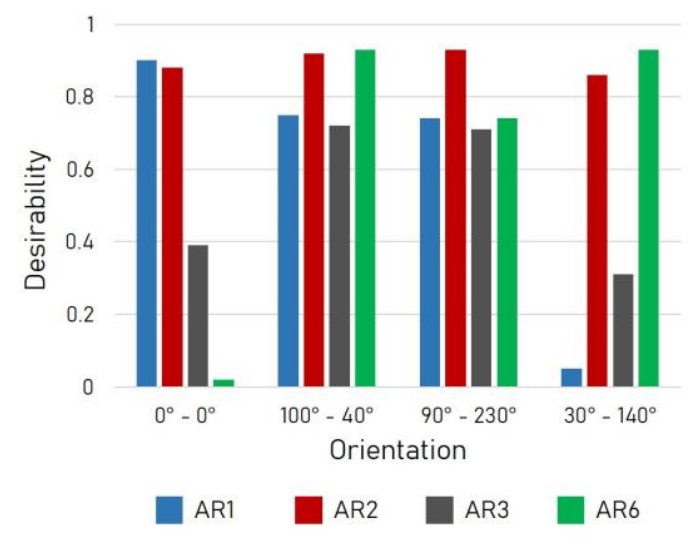

Figure 10. Example of orientation desirability comparison

\subsection{Benchmark}

The optimum desirability proposed by Magics is reported on the 3D responses to find the associated desirability. Only supported surfaces (equivalent to AR2) and projection on start plate (equivalent to AR1) are considered. By assigning a weight of 50\% to each criterion, the orientation found by Magics is the pair $\alpha=45^{\circ}-\beta=45^{\circ}$ for which the desirability values are 0.73 and 0.93 for respectively the projection on the start plate (AR1) and the overhang surfaces (AR2). This orientation is a trade-off between action rules. It is generally noticed that the results from both tools are aligning. However, the desirability approach allows finding more alternatives. For instance, the orientation $\alpha=90^{\circ}-$ $\beta=320^{\circ}$ results in the desirability values $D_{A R 1}=0.81$ and $D_{A R 2}=0.82$ which would constitute a better balancing. Moreover, the proposed approach facilitates the decision making process as it provides comparable metrics.

\section{CONCLUSION}

This paper addressed the problem of part orientation in powder bed fusion additive manufacturing. A review has revealed a set of action rules used by industry experts to ensure the good manufacturing of their parts. The methodology proposed here consisted of assisting the decision-making about orientation parameters based on company expertise. Using a knowledge mathematization approach, 
metrics representing the level of compliance with action rules have been proposed to facilitate the comparison of conformity between orientations. The complex shaped hammock support presented in the case study showed that this approach offers the possibility to compare several manufacturing scenarios based on quantitative values. Instead of proposing optimum points, multiple alternatives are offered to the decision-maker so that he is able to make trade-offs between his objectives.

As a future work, the re-design process of parts in fixed orientation can be foreseen based on the desirability values. The main idea is to attach to each surface of the part a desirability value, and those with a low value undergo shape optimization until their desirability is sufficiently acceptable.

\section{ACKNOWLEDGMENTS}

This work benefited from the support of the project COFFA ANR-17-CE10-0008 of the French National Research Agency (ANR) and DP Research Institute KAM Lab project.

\section{REFERENCES}

Cooke, N. J., (1994). "Varieties of Knowledge Elicitation Techniques". International Journal HumanComputer Studies 41, 801-849.

Costa, N. R., Lourenço, J., and Pereira, Z. L. (2011). "Desirability Function Approach: A Review and Performance Evaluation in Adverse Conditions". Chemometrics and Intelligent Laboratory Systems 107, 234-244.

Das, P., Chandran, R., Samant, R. and Anand, S. (2015). "Optimum part build orientation in additive manufacturing for minimizing part errors and support structures”. Procedia Manufacturing 1, 343-354.

Delfs, P., Tows, M. and Schmid, H.-J. (2016). "Optimized build orientation of additive manufactured parts for improved surface quality and build time”. Additive Manufacturing 12, 314-320.

Derringer, G. and Suich, R. (1980). "Simultaneous Optimization of Several Response Variables". Journal of Quality Technology 12(4), 214-219.

Gao, J., Zheng, D.T. and Gindy, N. (2004). "Extraction of machining features for CAD/CAM integration". The International Journal of Advanced Manufacturing Technology 24, 573-581.

Ghaoui, S., Ledoux, Y., Vignat, F., Museau, M., Vo, T. H., Villeneuve, F. and Ballu, A. (2020). “Analysis of geometrical defects in overhang fabrications in electron beam melting based on thermomechanical simulations and experimental validations". Additive Manufacturing 36.

Grandvallet, C., Mbow, M. M., Mainwaring, T., Vignat, F., Pourroy, F., and Marin, P. R. (2020). "Eight Action Rules for the Orientation of Additive Manufacturing Parts in Powder Bed Fusion: an Industry Practice". International Journal on Interactive Design and Manufacturing 14, 1159-1170.

Körner, C. (2016). "Additive manufacturing of metallic components by selective electron beam melting — a review”, International Materials Reviews 61, 5, 361-377.

Kranz, J., Herzog, D. and Emmelmann, C. (2015). "Design Guidelines for Laser Additive Manufacturing of ightweight Structures in TiAl6V4". Journal of Laser Applications 27.

Kumar, S., Singh, R., and Sekhon, G.S., "CCKBS: A component check knowledge-based system for assessing manufacturability of sheet metal parts", Journal of Materials Processing Technology 172, 64-69.

Hussein, A., Hao L., Yan, C., Everson, R. and Young, P. (2013). Advanced Lattice Support Structures for Metal Additive Manufacturing. Journal of Materials Processing Technology 213, 1019-1026.

Leutenecker-Twelsiek, B., Klahn, C. and Meboldt, M. (2016). "Considering Part Orientation in Design for Additive Manufacturing", Procedia CIRP 50, 408-413.

Mbow, M. M., Grandvallet, C., Vignat, F., Marin, P. R., Perry, N. and Pourroy, F. (2021). "Mathematization of Experts Knowledge: Example of part orientation in additive manufacturing". Journal of Intelligent Manufacturing.

Mugwagwa, L., Dimitrov, D., Matope, S., and Yadroitsev, I. (2018). "Influence of process parameters on residual stress related distortions in selective laser melting". Procedia Manufacturing 21, 92-99.

Sanfilippo, E. M., Belkadi, F. and Bernard, A. (2019). "Ontology-based knowledge representation for additive manufacturing”. Computers in Industry 109, 182-194.

Vayre, B. (2014). "Design for Additive Manufacturing, focus on EBM technology”, Phd thesis, Génie des procédés. Université de Grenoble. Français. 〈NNT : 2014GRENI096〉. 〈tel-01304269〉.

Vo, T. H., Museau, M., Vignat, F., Villeneuve, F., Ledoux, Y. and Ballu, A. (2018). "Typology of geometrical defects in Electron Beam Melting”. Procedia CIRP 75, 92-97.

Zhang, Y., Bernard, A., Harik, R. and Karunakaran, K.P (2017). "Build orientation optimization for multi-part production in additive manufacturing”. Journal of Intelligent Manufacturing 28, 1393-1407. 